\title{
AVALIAÇÃO DO PH SALIVAR EM INDIVÍDUOS DE DIFERENTES FAIXAS ETÁRIAS
}

\author{
João Armando Brancher \\ Pontifícia Universidade Católica Do Paraná - PUC/PR \\ Universidade Positivo - UP \\ brancher.a@pucpr.br
}

Brunah de Oliveira Buche Universidade Positivo - UP brunahbuche@hotmail.com

Ana Paula de Lima Silva Universidade Positivo - UP aninhawb@msn.com

Sidnei De Souza Chamberlain Universidade Federal Do Paraná - UFPR sidneichamberlain@hotmail.com

Maria Fernanda Torres Universidade Federal Do Paraná - UFPR torres.fernanda88@gmail.com

Ana Tereza Bittencourt Guimarães

Universidade Federal Do Paraná - UFPR anat@brturbo.com.br

\section{Resumo}

A saliva é um importante fluido bucal que contém um grande número de constituintes orgânicos e inorgânicos que promovem a proteção dos tecidos bucais. Ela é importante para a preservação e manutenção da saúde bucal, embora ainda receba pouca atenção por parte dos clínicos. O objetivo deste estudo foi mensurar o $\mathrm{pH}$ salivar em uma população bem caracterizada de indivíduos residentes em Curitiba, Brasil. Materiais e Métodos: A saliva total estimulada foi coletada de 536 indivíduos não aparentados em diferentes faixas etárias: Gi. 0-10 (n=91); Gii. 11-20 ( $\mathrm{n}=196)$; Giii. 21-59 (n=195) e Giv. 60-75 ( $\mathrm{n}=54)$ anos de idade. 
As amostras foram analisadas em um pHmetro digital da marca Micronal@. Durante a coleta de saliva, os indivíduos receberam instruções de higiene bucal. Resultados: Os valores de $\mathrm{pH}$ observados em cada grupo foram: Gi. 7.54 ( \pm 0.32$)$; Gii. $7.45( \pm 0.34)$; Giii. $7.30( \pm 0.40)$ e Giv. $7.08( \pm 0.42)$, respectivamente. Estes resultados mostram um decréscimo significativo no $\mathrm{pH}$ bucal com o avanço da idade ( $p>0,05$ ). Conclusão: Houve um decréscimo estatisticamente significativo no valor do $\mathrm{pH}$ salivar nos indivíduos estudados, especialmente naqueles com mais de 60 anos de idade.

Palavras-chave: Educação em saúde bucal. Idosos. Saliva.

\title{
SALIVARY PH RATES IN INDIVIDUALS OF DIFERENT AGES
}

\begin{abstract}
Saliva is a most valuable oral fluid that contains a large number of organic and inorganic constituents which promote the protection of the oral tissues. It is important to the preservation and maintenance of oral health, however yet it still receives little attention by the clinicians. The aim of this study was to measure the salivary $\mathrm{pH}$ in a well-characterized sample of individuals in Curitiba, Brazil. Method: Stimulated whole saliva was collected from 536 unrelated individuals in different age groups: i. 0-10 $(n=91)$; ii. 11-20 $(n=196)$; iii. 21-59 $(\mathrm{n}=195)$ and iv. 60-75 $(\mathrm{n}=54)$ years old.The samples were analyzed for $\mathrm{pH}$ with a $\mathrm{pHmeter}$ Micronal ${ }^{\circledR}$. During the collection of saliva, subjects received instructions on oral hygiene. Result: The mean values of $\mathrm{pH}$ observed were: i. $7.54( \pm 0.32)$; ii. $7.45( \pm 0.34)$; iii. 7.30 $( \pm 0.40)$ and iv. $7.08( \pm 0.42)$, respectively. These results show a decrease in oral $\mathrm{pH}$ in individuals with advancing age ( $>>0.05)$. Conclusion: There were significant differences in salivary $\mathrm{pH}$ in the subjects studied.
\end{abstract}

Keywords: Education in oral health. Elderly. Saliva 


\section{INTRODUÇÃOO}

A cavidade bucal é um ecossistema complexo e facilmente influenciável por mudanças ambientais. A progressão de doenças bucais tais como cárie dentária e doenças periodontais, entre outras, depende do frágil equilíbrio entre eventos que ocorrem na boca (UITTO et al, 2012).

A complexidade etiológica das doenças bucais é indiscutível. Alguns fatores são determinantes para a iniciação destas doenças, assim como microbiota bucal em desequilíbrio (NOBRE DOS SANTOS et al, 2002), má-higiene (PETERSEN, 2003) e hábitos inadequados levam a formação de colônias bacterianas na boca, denominadas de biofilme bucal (ZIJINGE et al, 2010).

Biologicamente, o biofilme bucal é determinante para o início e progressão das mais variadas doenças (PETERSEN, 2003). Nesse microambiente, a saliva desempenha um papel importante, pois o fluxo, a capacidade tampão, $\mathrm{pH}$ e a composição salivar podem impedir a progressão de doenças bucais (VAN NIEUW AMERONGEN et al, 2004). Ela contém uma série de constituintes inorgânicos e orgânicos os quais mantêm a saúde dos tecidos bucais, contribuindo também para a manutenção da saúde geral do indivíduo (UITTO et al, 2012).

Mesmo sendo o principal fluido protetor dos tecidos bucais, a saliva ainda recebe pouca atenção por parte dos profissionais da área de saúde. Trata-se de um fluido único e, por isso, é interessante como um método de diagnóstico de doenças bucais e sistêmicas, assim como os exames hematológicos (STRECKFUS, BIGLER, 2002; SAMARANAYAKE, 2007;), entretanto com vantagens adicionais uma vez que o acesso é fácil e a coleta é não invasiva. Apesar disso, sua falta só é percebida quando há uma acentuada redução no seu volume, na sua capacidade de tamponamento ou no seu pH (FEJERSKOV, 2004).

$\mathrm{Na}$ última década também foram reconhecidos como fatores que interagem e contribuem para o desenvolvimento de doenças bucais os fatores de risco genéticos (WERNECK et al, 2010), ambientais e comportamentais (SUSIN et al., 2004).

No caso de fatores comportamentais, a informação às pessoas é fundamental, uma vez que são responsáveis pela mudança de hábitos, muitos deles, deletérios. Pensando nisso, atividades de extensão universitária vem sendo realizadas permanentemente por acadêmicos de Odontologia nas Unidades de Saúde (US), na cidade de Curitiba.

Neste estudo, atividades lúdicas foram desenvolvidas por estudantes de odontologia com o propósito de levar informações sobre educação em saúde bucal aos indivíduos 
residentes no bairro do Pilarzinho, em Curitiba - Paraná. Além disso, o objetivo foi mensurar o pH salivar em uma amostra bem caracterizada de indivíduos em diferentes faixas em diferentes faixas etárias atendidos naquela US.

\section{MATERIAL E MÉTODOS}

\section{Aspectos Éticos, Sociais e Comportamentais}

A pesquisa foi desenvolvida na Unidade de Saúde (US) Vista Alegre, distrito Sanitário do Boa Vista, em Curitiba/PR entre os meses de julho e outubro de 2012. Alunos do curso de Odontologia dirigiram-se à esta US e, enquanto os indivíduos esperavam por atendimento, convidaram os mesmos para assistir à palestras sobre educação em saúde bucal enfatizando técnicas de escovação, utilização de fio dental, hábitos alimentares, entre outros.

Após as palestras, os indivíduos foram informados sobre o objetivo da pesquisa e convidados a participar da doação voluntária de saliva. Foram incluídos no estudo indivíduos que assinaram os termos de consentimento livre e esclarecido. No caso de menores de idade, pais ou responsáveis autorizaram a participação do menor na pesquisa, sempre obedecendo aos requisitos estabelecidos pelo Comitê de Ética em Pesquisa da Prefeitura Municipal de Curitiba, protocolo número 37/2012, conforme a Resolução 196/96 do Conselho Nacional de Saúde.

Todo e qualquer indivíduo que apresentasse problemas de saúde bucal foi encaminhado ao serviço de atendimento odontológico da própria prefeitura ou de universidades próximas àquela US. Ao todo, aproximadamente 2000 pessoas receberam orientações sobre educação em saúde bucal.

\section{Seleção do Grupo Amostral}

Para a amostra ser representativa, o cálculo estatístico do número amostral foi realizado assumindo um erro amostral de $4,1 \%$ e com as proporções de cada uma das classes etárias obtidas para o distrito como um todo.

Assumindo 95\% de confiança e erro amostral de $5 \%$, o número de indivíduos selecionados, nas diferentes faixas etárias, foi de quinhentos e trinta e seis (536) pessoas nãoaparentadas, de ambos os sexos, com idades variando entre 5 e 80 anos residentes no bairro 
do Pilarzinho que foram divididos em 04 grupos, dependendo da faixa etária: G-i. 5-10 anos ( $\mathrm{n}=91)$; G-ii. 11-20 anos (n=196); G-iii. 21-59 anos ( $\mathrm{n}=195)$ e G-iv. 60-80 anos ( $\mathrm{n}=53)$.

\section{Coleta da Saliva}

A saliva total estimulada foi coletada de acordo com a metodologia descrita por Klock e Krasse (1977). Para a coleta da amostra, cada indivíduo sentou-se confortavelmente em uma cadeira comum e recebeu como estímulo mastigatório um pedaço de mangueira de látex, esterilizado com $1 \mathrm{~cm}$ de comprimento por $0,5 \mathrm{~cm}$ de diâmetro preso a $20 \mathrm{~cm}$ de fío dental para evitar que seja deglutido. O voluntário mastigou a mangueira de látex e toda a saliva produzida foi depositada em um frasco coletor previamente esterilizado. $\mathrm{O} \mathrm{pH}$ salivar foi mensurado imediatamente após a coleta, com o auxílio de um potenciômetro da marca Metler Toledo®, segundo recomendações do fabricante.

\section{Análise Estatística}

Os dados referentes ao $\mathrm{pH}$ salivar de 536 pessoas de diferentes classes etárias foram analisados quanto ao padrão de distribuição por meio do teste de Lilliefors. Uma vez que os dados se encontravam em normalidade, assim como se encontravam em homocedasticidade, as médias de $\mathrm{pH}$ de cada classe etária foram comparadas por meio do teste de ANOVA-fator único. Em caso de significância estatística, os dados foram comparados por meio do teste de acompanhamento de Tukey para amostras desiguais.

\section{RESULTADOS E ANÁLISE}

Ações preventivas de educação em saúde bucal devem ser constantes e frequentes. Alguns estudos mostram que a prevalência de doenças bucais tem reduzido significativamente, inclusive na América Latina e no Brasil (BONECKER, CLEATONJONES, 2003; MULLEN, et al, 2012). A explicação para essa redução deve-se, possivelmente, à ação de programas de saúde bucal e a mudanças comportamentais dos indivíduos frente às informações recebidas (SUSIN et al., 2004).

Percebe-se desta maneira que o acesso à informação, por parte dos indivíduos, é extremamente importante. Nesta pesquisa acadêmicos de Odontologia desenvolveram atividades de extensão universitária na US Vista Alegre, em Curitiba, Paraná. Ações de 
promoção em saúde bucal a pacientes que aguardavam atendimento foram executadas. Os indivíduos, em pequenos grupos, assistiram a vídeos educativos, receberam escovas dentais, dentifrícios e orientações de higiene e saúde bucal. Mães, pais ou responsáveis por menores de idade foram orientados e motivados no sentido de estimular os seus filhos para adoção de hábitos adequados de saúde bucal.

Cerca de 2000 pessoas, de diferentes idades, assistiram palestras e tiveram acesso a informações básicas sobre prevenção de doenças bucais e orientações sobre os procedimentos a serem adotados quando são acometidos por alguma patologia bucal. As atividades foram desenvolvidas no período compreendido entre os meses de julho e outubro de 2012.

É importante destacar que o tratamento de doenças bucais é oneroso. A cárie dentária, por exemplo, nos países de terceiro mundo, representa a quarta doença que mais dispende gastos por parte dos governos (YEE, SHEIHAM, 2002). Outros estudos mostram que em ambiente hospitalar, o acúmulo de biofilme bacteriano, especialmente em pacientes internados em unidades de terapia intensiva, é responsável por complicação ou evolução de doenças sistêmicas pré-existentes. Neste caso específico o treinamento de profissionais da área de Enfermagem é indispensável.

Como o propósito deste estudo também foi avaliar o $\mathrm{pH}$ salivar nesta população, os indivíduos receberam informações sobre a importância da saliva na cavidade bucal e a relação desta com situações de estresse emocional, alterações imunológicas e neurológicas, além do estado nutricional e metabólico do indivíduo. Também foram informados sobre as funções locais da saliva e sobre como a saliva pode ser utilizada como recurso diagnóstico inclusive substituindo os exames hematológicos. Desta forma, 536 indivíduos concordaram em doar voluntariamente saliva. As características dessa população são mostradas na tabela 1 .

\begin{tabular}{|c|c|c|c|c|c|}
\hline Classes etárias & $\mathrm{N}$ & Média de $\mathrm{pH}$ & Desvio Padrão & Erro Padrão & IC $95 \%$ \\
\hline $5-10$ anos & 91 & $\begin{array}{c}7,54(\mathrm{a}) \\
(6,6-8,4)\end{array}$ & 0,33 & 0,034 & $7,47-7,61$ \\
\hline $11-20$ anos & 196 & $\begin{array}{c}7,46(a) \\
(6,5-8,7)\end{array}$ & 0,35 & 0,025 & $7,41-7,51$ \\
\hline $21-59$ anos & 195 & $\begin{array}{c}7,30(b) \\
(5,7-8,2)\end{array}$ & 0,40 & 0,029 & $7,24-7,36$ \\
\hline $60-80$ anos & 53 & $\begin{array}{c}7,09(\mathrm{c}) \\
(6,1-7,7)\end{array}$ & 0,43 & 0,059 & $6,97-7,21$ \\
\hline
\end{tabular}


A literatura mostra que a saliva desempenha papel importantíssimo na manutenção da saúde bucal pois apresenta vários fatores inatos e adquiridos capazes de inibir a invasão, crescimento e metabolismo bacteriano por meio de diferentes mecanismos (VAN NIEUW AMERONGEN, VEERMAN, 2002; KIDD, FEJERSKOV, 2004). Pode modular a adesão de bactérias aos dentes e atenuar os efeitos deletérios da produção de metabólitos por parte da microbiota bucal (TENOVUO, 2002). Por isso, pesquisadores tem investigado o papel da saliva na evolução das doenças bucais (BURT, PAI, 2001; SINGH et al, 2002), especialmente a quantidade de saliva produzida, denominada fluxo salivar, a capacidade de tamponamento salivar e o pH salivar (KIDD, FEJERSKOV, 2004).

O fluxo salivar é uma mensuração muito individualizada e varia de acordo com o ritmo circadiano, mas sabe-se que fluxo constante de saliva pode eliminar eficientemente microorganismos da cavidade bucal, e fluxo reduzido pode facilitar o crescimento bacteriano (ZIJINGE et al, 2010).

Capacidade de tamponamento salivar é a capacidade que a saliva possui de manter o pH bucal estável, dentro dos limites de normalidade, ou seja, é a capacidade que a saliva possui de neutralizar ácidos e ou bases presentes na cavidade bucal contribuindo para a saúde bucal (CHEAIB, LUSSI, 2013).

Com relação ao $\mathrm{pH}$ salivar, objeto de estudo nesta pesquisa, sabe-se que o mesmo sofre alterações após o consumo de bebidas ou alimentos sólidos e depende também do fluxo e da capacidade tampão da saliva. $\mathrm{O}$ pH da saliva mostra a presença ou não de ácidos na boca. Qualquer alteração nos valores de $\mathrm{pH}$, para baixo ou para cima, pode ser responsável pela iniciação e evolução de doenças bucais. Há um consenso na literatura de que o pH bucal, em média, varia entre 6,8 e 7,2 nas diferentes populações do mundo, com pequenas alterações para baixo ou para cima, independente da idade (KIDD, FEJERSKOV, 2004).

Este estudo avaliou o $\mathrm{pH}$ salivar de indivíduos em diferentes faixas etárias atendidos na US Vista Alegre, em Curitiba, Paraná. Foram selecionadas 536 pessoas não aparentadas de ambos os sexos cuja idade variou entre 5 e 80 anos. Foi possível observar que as médias de pH das classes etárias de 0 a 10 anos e 11 a 20 anos foram consideradas estatisticamente equivalentes entre si $(\mathrm{p}>0,05)$. Contudo, estas duas primeiras classes etárias apresentaram significativamente maiores médias quando comparadas às demais $(\mathrm{p}<0,05)$ (Tabela 1). As classes etárias de 21 a 59 anos e acima de 60 anos apresentaram médias estatisticamente diferentes das demais $(\mathrm{p}<0,05)$ (Figura. 1).Como pode ser observado na figura 1, há um 
decréscimo significativo no valor do $\mathrm{pH}$ salivar em função do aumento da idade. Estes resultados mostram um evidente decréscimo no $\mathrm{pH}$ bucal dos indivíduos com o avançar da idade ( $p>0.05$ ) embora o menor valor de $\mathrm{pH}$ observado tenha sido 5,7 observado em um indivíduo do grupo cuja idade variou entre 21 e 59 anos.

Os resultados obtidos neste estudo para $\mathrm{pH}$ salivar foram ainda menores do que os valores observados em outros estudos similares (JOHANSSON et al, 1994; MAZENGO et al, 1994) porém estão dentro dos limites descritos na literatura (FEJERSKOV, 2004).

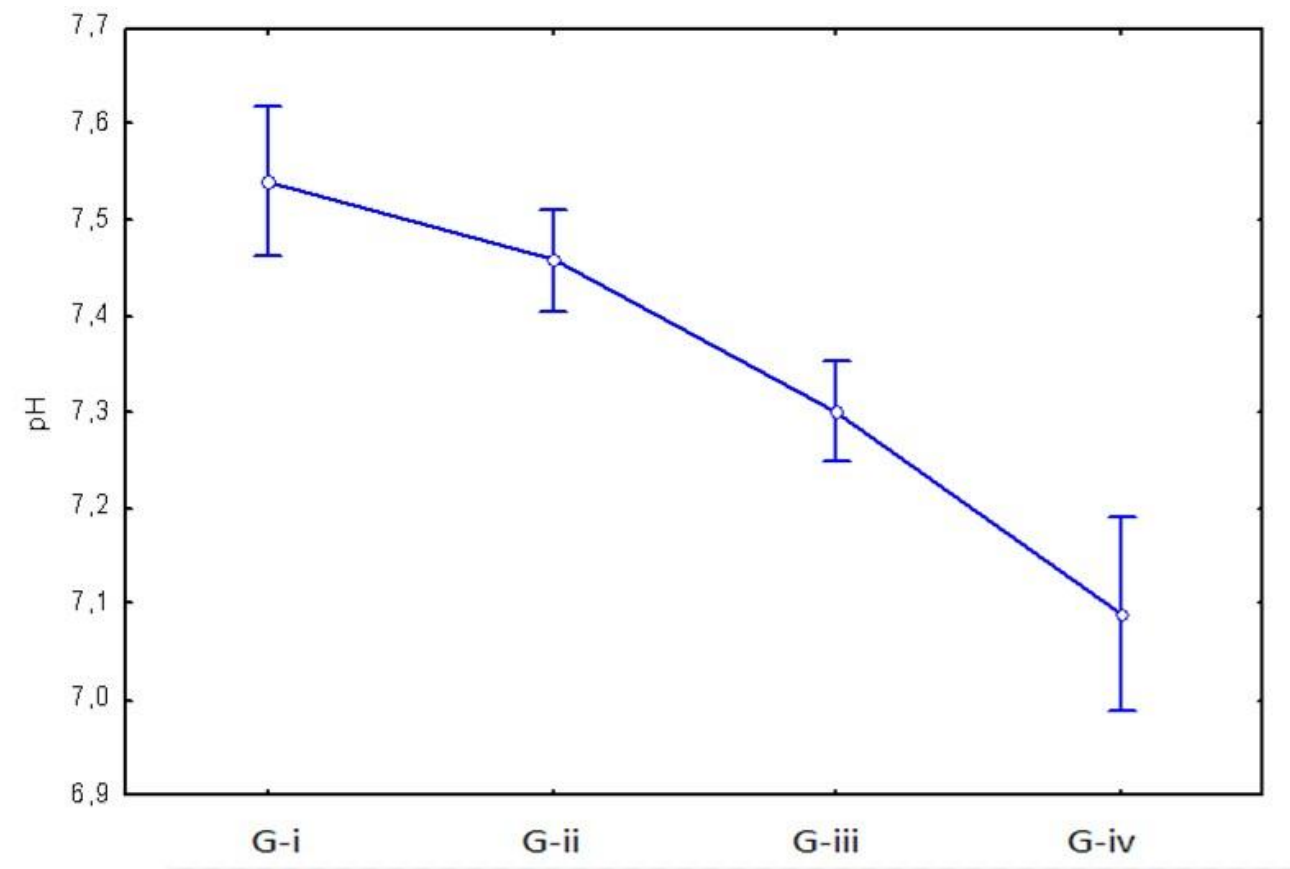

Figura 1- Valores de pH salivar observados nos diferentes grupos.

Fonte: Elaborado pelos autores:

Sabe-se que um volume constante de saliva na boca contribui decisivamente para a manutenção do $\mathrm{pH}$ salivar. Também é sabido que o fluxo salivar decresce com o aumento da idade (KIDD, FEJERSKOV, 2004), o que implica na diminuição do pH bucal. Neste caso o estímulo para produção de saliva é importante. Na população em geral, mastigar auxilia decisivamente na digestão e absorção dos alimentos proporcionando uma boa nutrição. Menos lembrado, porém tão importante quanto, é o fato de que a mastigação promove maior atividade muscular dos lábios, músculos da mastigação e língua com isso estimulando a produção de saliva e manutenção do $\mathrm{pH}$ salivar dentro dos limites de normalidade. 


\section{CONSIDERAÇÕES FINAIS}

As atividades desenvolvidas neste estudo confirmam dados já conhecidos da literatura: acesso à informação é indispensável. No início da pesquisa os acadêmicos de Odontologia preocuparam-se com os questionamentos que receberiam dos indivíduos. Com o decorrer do tempo, perceberam que muitas dúvidas eram simples, facilmente respondidas. A assimilação, por parte dos voluntários foi satisfatória.

Sobre o pH salivar, embora não existam dados definitivos sobre a sua variação em diferentes populações, neste estudo observou-se um decréscimo significativo no valor deste com o avançar da idade. Como existe uma relação direta entre o volume de saliva produzida e o pH, ações educativas futuras visarão estimular o fluxo salivar na população mais idosa.

\section{REFERÊNCIAS}

BONECKER, Marcelo, CLEATON-JONES, Peter. Trends in dental caries in Latin American and Caribbean 5-6- and 11-13-year-old children: a systematic review. Community Dental Oral Epidemiology, v. 31, p. 152-157, 2003.

BURT, Brian A, PAI, Satishchandra. Sugar consumption and caries risk: a systematic review. Journal of Dental Education, v. 65, p. 1017-1027, 2001.

CHEAIB, Zeinab, LUSSI, Adrian. Role of amylase, mucin, IgA and albumin on salivary protein buffering capacity: A pilot study. Journal of Biosciences, v.38, p. 259-265, 2013.

DAWES, Colin, KUBIENIEC, Karl. The effects of prolonged gum chewing on salivary flow rate and composition. Archives Oral Biology, v. 49, p. 665-669, 2004.

FEJERSKOV, Ole. Changing paradigms in concepts on dental caries: consequences for oral health care. Caries Research, v. 38, p. 182-189, 2004.

JENTSCH Holger, BEETKE Eckhard, GOCKE Robert. Salivary analyses and caries increment over 4 years an approach by cluster analysis. Clinical Oral Investigation, v. 8, p. 156-160, 2004.

JOHANSSON, Ingegerd, LENANDER-LUMIKARI, Marianne, SAELLSTRÖM Arnold K. Saliva composition in Indian children with chronic protein-energy malnutrition. Jounal Dental $\begin{array}{llllll}\text { Research, } & \text { v. } & 1, & \text { p. } & 11-19, & 1994 .\end{array}$ 
KIDD, Edwina A, FEJERSKOV, Ole. What constitutes dental caries? Histopathology of carious enamel and dentin related to the action of cariogenic biofilms. Journal of Dental Research, v. 83, p. 35-38, 2004.

KLOCK, Bjorn, KRASSE, Bo. Microbial and salivary conditions in 9- to 12-year-old children. Scandinavian Journal Dental Research, v. 85, p. 56-63, 1977.

MANDEL, Irwin D. Impact of saliva on dental caries. Compendium of Continuing Education in Dentistry, v.1, p. 476-481, 1989.

MULLEN, Joe, MCGAFFIN, Judi, FARVADIN, Nader, BRIGHTMAN, Stephen, HAIRE, Carl, FREEMAN, Robert. Caries status in 16 year-olds with varying exposure to water fluoridation in Ireland. Community Dental Health, v. 29, p. 293-296, 2012.

MAZENGO, Marc C, TENOVUO, Jorma, SIMEL, Olli, et al. Flow rate and composition of whole saliva in rural and urban Tanzania with special reference to diet, age, and gender.Caries Research, v. 28, p. 468-476, 1994.

NOBRE DOS SANTOS, Marinês, MELO DOS SANTOS, Luís, CURY, Jayme A. Relationship among dental plaque composition, daily sugar exposure and caries in the primary dentition. Caries Research, v. 36, p. 347-352, 2002.

PETERSEN, Poul Erik. The World Oral Health Report 2003: continuous improvement of oral health in the 21 st century - the approach of the WHO Global Oral Health Programme. Community Dentistry and Oral Epidemiology, v. 31, p, 3-24, 2003.

SAMARANAYAKE, Lakshman. Saliva as a diagnostic fluid. International Dental Journal, v. 57, p. 295-299, 2007.

SINGH, Pradhyumna K, PARSEK, Matthew, et al. A component of innate immunity prevents bacterial biofilm development. Nature, v. 417, p 552-555, 2002.

STRECKFUS, Charles F, BIGLER, Leonora R._Saliva as a diagnostic fluid. Oral Disease, v. 8, p. 69-76, 2002.

SUSIN, Cristiano, OPPERMANN, Rui, HAUGEJORDEN, Ola, ALBANDAR, Jasim. Periodontal attachment loss attributable to cigarette smoking in an urban Brazilian population.

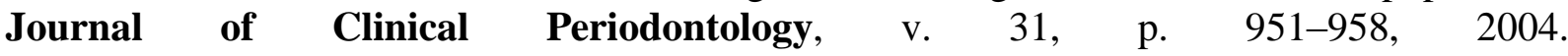


TENOVUO, Jorma. Clinical applications of antimicrobial host proteins lactoperoxidase, lysozyme and lactotransferrin in xerostomia: efficacy and safety. Oral Diseases, v.8, p. 2329, 2002.

UITTO, Veli Jukka, NYLUND Karl, PUSSINEN, Pirkko. The association of oral microbiota and general health. Duodecm, v. 128, p. 1232-1237, 2012.

VAN NIEUW AMERONGEN, Arie, BOLSCHER, Jan, VEERMAN, Enno C. Salivary proteins: protective and diagnostic value in cariology? Caries Research, v.38, p. 247-253, 2004.

VAN NIEUW AMERONGEN, Arie, VEERMAN Enno C. Saliva - The defender of oral cavity. Oral Diseases, v. 8, p. 12-22, 2002.

YEE, Robert, SHEIHAM, Aubrey. The burden of restorative dental treatment for children in third world contries. International Dental Journal, v. 52, p. 1-0, 2002.

WERNECK, Renata Iani, MIRA, Marcelo, TREVILlATTO, Paula Cristina. A critical review: an overview of genetic influence on dental caries. Oral Diseases, v. 16, p. 613-623, 2010 .

ZIJINGE, Vicent, VAN LEEUWEN, Barbara, DEGENER John, ABBAS, Frank, THURNHEER, Thomas et al. Oral Biofilm Architecture on Natural Teeth, v. 5, p. 1-9, 2010. 